Study of hall current, radiation and velocity slip on hydromagnetic physiological hemodynamic fluid with porous medium through joule heating and mass transfer in presence of chemical reaction

\title{
Ravikumar Seelam
}

Department of Mathematics, NBKR Institute of Science and Technology (An Autonomous Institution, Accredited by NBA and A-Grade of NAAC, ISO 9001:2008 Certified), Vidyanagar, SPSR Nellore 524413, Andhra Pradesh, India

Corresponding Author Email:drsravikumar1979@gmail.com

https://doi.org/10.18280/ijht.360206

Received: 8 October 2017

Accepted: 3 May 2018

Keywords:

chemical reaction, hall current, porosity parameter, joule heating, mass transfer radiation

\begin{abstract}
In this present paper, the problem of hall current, radiation and velocity slip on hydromagnetic physiological hemodynamic fluid with joule heating and mass transfer in presence of chemical reaction in an inclined asymmetrical tapered vertical channel have been investigated. An analysis has been carried out in the presence of the porous medium. Analytical solution is carried out under long wavelength and low-reynolds number approximations. Numerical results were presented for axial pressure gradient, temperature, concentration, nusselt number $(\mathrm{Nu})$ and sherwood number $(\mathrm{Sh})$. Variations of the said quantities with dissimilar parameters are computed by using MATHEMATICA software. Graphs reflecting the contributions of embedded parameters were discussed. It is worth mentioning that the pressure gradient $(\mathrm{dp} / \mathrm{dx})$ enhances with an increase in hartmann number $(M)$, gravitational parameter $(\eta)$ and slip parameter $(\beta)$ while it reduces by an increase in porosity parameter $(\mathrm{Da})$, hall parameter $(\mathrm{m})$ and volumetric flow rate $(\bar{Q})$. We notice that the temperature of the fluid rises with an increase in hartmann number $(\mathrm{M})$, porosity parameter $(\mathrm{Da})$, radiation parameter $(\mathrm{N})$, prandtl number $(\mathrm{Pr})$, brinkman number $(\mathrm{Br})$, heat source parameter $(v)$ and hall current parameter $(\mathrm{m})$. We observe that the result in concentration profile reduces with a rise in chemical reaction parameter.
\end{abstract}

\section{INTRODUCTION}

It is commonly accepted now that peristaltic flows are instigated by the propagation of waves along the flexible boundaries of channel or tube. Such flows in physiology are exemplified by food movement in the digestive tract, urine transport from kidney to bladder, semen movement in vas deferens, movement of lymphatic fluids in lymph vessels, bile flow from the gallbladder into the duodenum, vasomotion of blood cells, movement of ovum in the female fallopian tube and transport of spermatozoa in the ductus efferents. Peristaltic motion in the industrial applications is employed in the transport of corrosive and noxious fluids, roller and finger pumps, hose pumps, tube pumps, dialysis machines and heart-lung machines. Latham [1] and Shapiro et al. [2] discussed the peristalsis of viscous fluids through theoretical and experimental approaches. Afterwards, a wealth of literature related to peristaltic pumping of viscous and non-Newtonian fluids exists in view of one or more assumptions of long wavelength, low reynolds number, small wave number and small amplitude ratio. Few recent investigations in this direction may be mentioned by the following references like Abd Elmaboud and Mekheimer [3], Mekheimer et al. [4-5], Hayat et al. [6], Pandey and Tripathi [7] and Tripathi et al. [8].

The interaction of peristalsis with heat and mass transfer has not received much attention. The thermodynamical aspects of blood may not be important when blood is inside the body but they become significant when it is drawn out of the body. Keeping in view the significance of heat transfer in blood flow, Srinivas and Kothandapani [9] analyzed the influence of heat and mass transfer on MHD peristaltic flow through a porous space with compliant walls. Lakshminarayana et al. [10] have reported the influence of heat transfer on MHD peristaltic flow through a vertical asymmetric porous channel. Vajravelu et al. [11] studied the influence of heat transfer on peristaltic transport of a jeffrey fluid in a vertical porous stratum. Agrawal [12] discussed the heat transfer to pulsatile flow of a conducting fluid through a porous channel in the presence of magnetic field. Tang et al. [13] studied the peristaltic flow of a heat conducting fluid subject to a prescribed pressure drop. Radhakrishnamacharya and Srinivasulu [14] studied the influence of wall properties on peristaltic transport with heat transfer. Having such preference in mind, some authors Abbasi et al. [15], Hayat et al. [16-17], Mekheimer et al. [18], Srinivas et al. [19], Eldabe et al. [20], Shehzad et al. [21], Ravikumar [22-24], Misra and Adhikary [25], Ravikumar and Abzal [26], Ameer Ahamad et al. [27] and Srinivasacharya and Shafeeurrahman [28] have analyzed the peristaltic transport with heat transfer.

The aim of the present research was to study the influence of hall current, radiation and velocity slip on hydromagnetic physiological hemodynamic fluid with joule heating and mass transfer in presence of chemical reaction. The momentum, temperature and concentration equations have been linearized under long wavelength and low-reynolds number assumptions and analytical solutions for the flow variables have been derived. Numerical values of the said quantities are presented graphically. The present study may be beneficial in understanding the dynamics of blood flow in 
blood vessels by taking into account of joule heating and mass transfer in presence of chemical reaction.

\section{FORMULATION OF THE PROBLEM}

We consider the MHD (magnetohydrodynamics) peristaltic transport of an incompressible viscous fluid in a two-dimensional uneven inclined perpendicular tapered channel under the influence of porous medium. The joule heating, hall currents, radiation and slip boundary conditions were taken into the account. The left wall of the channel is maintained at temperature $T_{0}$ whereas the right wall is maintained at temperature $T_{1}$. We tend to assume that the fluid is subject to a relentless transverse magnetic field $\mathrm{B}_{0}$. The fluid is induced by sinusoidal wave trains propagating with constant speed $c$ along the channel walls.

The geometry of the wall deformations are drawn by the subsequent expressions

$Y=H_{2}=b+m^{I} X+d \sin \left[\frac{2 \pi}{\lambda}(X-c t)\right]$

$Y=H_{1}=-b-m^{I} X-d \sin \left[\frac{2 \pi}{\lambda}(X-c t)+\phi\right]$.

where $b$ is the half-width of the channel, $d$ is the wave amplitude, $c$ is the phase speed of the wave and $m^{I}$ is the non-uniform parameter, $\lambda$ is the wavelength, $\mathrm{t}$ is the time and $\mathrm{X}$ is the direction of wave propagation. The phase difference $\phi$ varies in the range $0 \leq \phi \leq \pi, \phi=0$ corresponds to the symmetric channel with waves out of phase and further $b, d$ and $\phi$ satisfy the following conditions for the divergent channel at the inlet $d \cos \left(\frac{\phi}{2}\right) \leq b$.

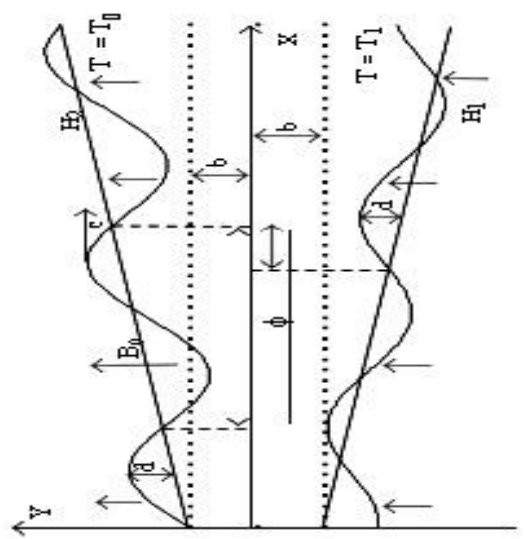

Figure 1. Schematic diagram of the physical model

The equations governing the motion for the present problem prescribed as follows [9, 29].

The continuity equation is

$\frac{\partial u}{\partial x}+\frac{\partial v}{\partial y}=0$

The momentum equations are

$$
\begin{aligned}
\rho\left[u \frac{\partial u}{\partial x}+v \frac{\partial u}{\partial y}\right]= & -\frac{\partial p}{\partial x}+u\left[\frac{\partial^{2} u}{\partial x^{2}}+\frac{\partial^{2} u}{\partial y^{2}}\right] \\
& +\left[\frac{\sigma B_{0}^{2}}{1+m^{2}}\right](m v-(u+c))
\end{aligned}
$$

$$
-\frac{\mu}{k_{1}}(u+c)+\rho g \sin \alpha,
$$

$$
\begin{aligned}
\rho\left[u \frac{\partial v}{\partial x}+v \frac{\partial v}{\partial y}\right]= & -\frac{\partial p}{\partial y}+u\left[\frac{\partial^{2} v}{\partial x^{2}}+\frac{\partial^{2} v}{\partial y^{2}}\right] \\
& -\left[\frac{\sigma B_{0}^{2}}{1+m^{2}}\right](m(u+c)+v) \\
& -\frac{\mu}{k_{1}}(v+c)-\rho g \cos \alpha,
\end{aligned}
$$

The energy equation is

$$
\begin{aligned}
\rho C_{p}\left[u \frac{\partial}{\partial x}+v \frac{\partial}{\partial y}\right] T= & k\left[\frac{\partial^{2}}{\partial x^{2}}+\frac{\partial^{2}}{\partial y^{2}}\right] T+Q_{0} \\
& +\sigma B_{0}^{2} u^{2}-\frac{\partial q}{\partial y},
\end{aligned}
$$

The concentration equation is

$$
\begin{aligned}
{\left[u \frac{\partial C}{\partial x}+v \frac{\partial C}{\partial y}\right] T=} & D_{m}\left[\frac{\partial^{2} C}{\partial x^{2}}+\frac{\partial^{2} C}{\partial y^{2}}\right]+\frac{D_{m} K_{T}}{T_{m}}\left[\frac{\partial^{2} T}{\partial x^{2}}+\frac{\partial^{2} T}{\partial y^{2}}\right] \\
& -k_{2}\left(C-C_{0}\right) .
\end{aligned}
$$

where $\mathrm{u}$ and $\mathrm{v}$ are the velocity components in the corresponding coordinates, $\mathrm{k}_{1}$ is the permeability of the porous medium, $\rho$ is the density of the fluid, $\mathrm{p}$ is the fluid pressure, $k$ is the thermal conductivity, $\mu$ is the coefficient of the viscosity, $Q_{0}$ is the constant heat addition/absorption, $C_{p}$ is the specific heat at constant pressure, $\sigma$ is the electrical conductivity, $g$ is the acceleration due to gravity and $\mathrm{T}$ is the temperature of the fluid.

The relative boundary conditions are

$U=0 \quad T=T_{0} \quad C=C_{0}$ at $Y=H_{1}$,

$U=0 \quad T=T_{1} \quad C=C_{1}$ at $Y=H_{2}$.

The radioactive heat flux [30] is given by

$\frac{\partial q}{\partial y}=4 \alpha^{2}\left(T_{0}-T_{1}\right)$

where $\alpha$ is the mean radiation absorption coefficient.

Introducing a wave frame $(\mathrm{x}, \mathrm{y})$ moving with velocity $\mathrm{c}$ away from the fixed frame $(\mathrm{X}, \mathrm{Y})$ by the transformation

$x=X-c t, y=Y, u=U-c, v=V$ and $p(x)=P(X, t)$

Introducing the following non-dimensional quantities [31]: $x^{*}=\frac{x}{\lambda}, y^{*}=\frac{y}{b}, t^{*}=\frac{c t}{\lambda}, u^{*}=\frac{u}{c}, v^{*}=\frac{v}{c \delta}, h_{1}=\frac{H_{1}}{b}, h_{2}=$ $\frac{H_{2}}{b}, \quad p^{*}=\frac{b^{2} p}{c \lambda \mu}, \quad \theta=\frac{T-T_{0}}{T_{1}-T_{0}}, \delta=\frac{b}{\lambda}, R e=\frac{\rho c b}{\mu}, \quad M=$ $B_{0} b \sqrt{\frac{\sigma}{\mu}}, \operatorname{Pr}=\frac{\mu C_{p}}{\kappa}, E_{c}=\frac{c^{2}}{C_{p}\left(T_{1}-T_{0}\right)}, v=\frac{Q_{0} b^{2}}{\mu C_{p}\left(T_{1}-T_{0}\right)}, N^{2}=$ $\frac{4 \alpha^{2} d^{2}}{k}, \varepsilon=\frac{d}{b}, \quad \Phi=\frac{C-C_{0}}{C_{1-} C_{0}}, \eta=\frac{\rho b^{2} g}{\mu c}, \eta_{1}=\frac{\rho b^{3} g}{\lambda \mu c}, S_{c}=\frac{\mu}{D_{m} \rho}$, $S_{r}=\frac{D_{m} \rho k_{T}\left(T_{1}-T_{0}\right)}{\mu T_{m}\left(C_{1}-C_{0}\right)}, S=\frac{K_{2} \rho d^{2}}{\mu}$.

where $\epsilon=\frac{d}{b}$ is the non-dimensional amplitude of channel, $\delta=\frac{b}{\lambda}$ is the wave number, $k_{1}=\frac{\lambda m^{I}}{b}$ is the non - uniform parameter, $\mathrm{Re}$ is the reynolds number, $\mathrm{M}$ is the hartmann number, $K=\frac{k}{b^{2}}$ is the permeability parameter, $\operatorname{Pr}$ is the prandtl number, $E_{c}$ is the eckert number, $v$ is the heat source/sink parameter, $g$ is the acceleration due to gravity, $C_{p}$ 
is the specific heat at constant pressure, $Q_{0}$ is the constant heat addition/absorption, $N^{2}$ is the radiation parameter, $\mathrm{B}_{\mathrm{r}}$ $\left(=\mathrm{E}_{\mathrm{c}} \mathrm{P}_{\mathrm{r}}\right)$ is the brinkman number, $\mathrm{D}_{\mathrm{m}}$ is the coefficient of mass diffusivity, $T_{m}$ is the mean temperature, $K_{T}$ is the thermal diffusion ratio, $\eta$ and $\eta_{1}$ are gravitational parameters, $S_{c}$ is the schmidt number, $S_{r}$ is the soret number and $S$ is the chemical reaction parameter.

\section{SOLUTION OF THE PROBLEM}

In view of the above transformations (9) and nondimensional variables (10), equations (3-7) are reduced to the following non-dimensional form after dropping the stars.

$\delta\left[\frac{\partial u}{\partial x}+\frac{\partial v}{\partial y}\right]=0$

$\operatorname{Re} \delta\left[u \frac{\partial u}{\partial x}+v \frac{\partial u}{\partial y}\right]=-\frac{\partial p}{\partial x}+\delta^{2} \frac{\partial^{2} u}{\partial x^{2}}+\frac{\partial^{2} u}{\partial y^{2}}$

$+\frac{M^{2}}{1+m^{2}}(m \delta v-(u+1))-\frac{1}{D} u-\frac{1}{D}+\eta \sin \alpha$

$R e \delta^{3}\left[u \frac{\partial v}{\partial x}+v \frac{\partial v}{\partial y}\right]=-\frac{\partial p}{\partial y}+\delta^{2}\left(\delta^{2} \frac{\partial^{2} v}{\partial x^{2}}+\frac{\partial^{2} v}{\partial y^{2}}\right)$

$-\frac{\delta M^{2}}{1+m^{2}}(m(u+1)+\delta v)-\delta^{2} \frac{1}{D} v-\delta^{2} \frac{1}{D}-\eta_{1} \cos \alpha$,

$R e\left[\delta u \frac{\partial \theta}{\partial x}+v \frac{\partial \theta}{\partial y}\right]=\frac{1}{P_{r}}\left[\delta^{2} \frac{\partial^{2} \theta}{\partial x^{2}}+\frac{\partial^{2} \theta}{\partial y^{2}}\right]+\beta$

$+M^{2} E_{c} u^{2}+\frac{N^{2} \theta}{P_{r}}$

$$
\begin{aligned}
\operatorname{Re} \delta\left[u \frac{\partial \phi}{\partial x}+v \frac{\partial \phi}{\partial y}\right]= & \frac{1}{S_{c}}\left[\delta^{2} \frac{\partial^{2} \phi}{\partial x^{2}}+\frac{\partial^{2} \phi}{\partial y^{2}}\right] \\
& +S_{r}\left[\delta^{2} \frac{\partial^{2} \theta}{\partial x^{2}}+\frac{\partial^{2} \theta}{\partial y^{2}}\right]-S \phi
\end{aligned}
$$

Applying long wavelength approximation and neglecting the wave number along with low-reynolds numbers. Equations (11 - 15) become

$\frac{\partial^{2} u}{\partial y^{2}}-\left(\frac{M^{2}}{1+m^{2}}+\frac{1}{D}\right) u=\frac{\partial p}{\partial x}+\left(\frac{M^{2}}{1+m^{2}}+\frac{1}{D}\right)-\eta \sin \alpha$,

$\frac{\partial p}{\partial y}=0$

$\frac{1}{P_{r}} \frac{\partial^{2} \theta}{\partial y^{2}}+v+M^{2} E_{c} u^{2}+\frac{N^{2} \theta}{P_{r}}=0$,

$\frac{1}{S_{c}} \frac{\partial^{2} \phi}{\partial y^{2}}+S_{r} \frac{\partial^{2} \theta}{\partial y^{2}}-S \phi=0$

The relative boundary conditions in dimensionless form are given by

$u=-\beta \frac{\partial u}{\partial y}, \theta=0, \phi=0$ at $y=h_{1}$,

$u=\beta \frac{\partial u}{\partial y}, \theta=1, \phi=1$ at $y=h_{2}$

where

$h_{1}=1-k_{1} x-\epsilon \sin [2 \pi(x-t)+\phi]$,

$h_{2}=1+k_{1} x+\epsilon \sin [2 \pi(x-t)]$.

The solutions of velocity and temperature with subject to boundary conditions (20) and (21) are given by

$u=A_{1} \sinh \left[\alpha_{1} y\right]+A_{2} \cosh \left[\alpha_{1} y\right]+B$

where

$A_{1}=-B a_{3}, \quad A_{2}=B a_{2}, \quad a_{2}=\frac{a_{4}}{a_{5}}, \quad a_{3}=\frac{a_{6}}{a_{7}}$,

$a_{4}=\left[\left[\sinh \left[\alpha_{1} h_{2}\right]-\beta \alpha_{1} \cosh \left[\alpha_{1} h_{2}\right]\right]-1\right]$

$a_{5}=-\left[\frac{\cosh \left[\alpha_{1} h_{1}\right]+\beta \alpha_{1} \sinh \left[\alpha_{1} h_{1}\right]}{\sinh \left[\alpha_{1} h_{1}\right]+\beta \alpha_{1} \cosh \left[\alpha_{1} h_{1}\right]}\right]$

$\times\left[\sinh \left[\alpha_{1} h_{2}\right]-\beta \alpha_{1} \cosh \left[\alpha_{1} h_{2}\right]\right]$ $+\left[\cosh \left[\alpha_{1} h_{2}\right]-\beta \alpha_{1} \sinh \left[\alpha_{1} h_{2}\right]\right]$,

$a_{6}=a_{2}\left[\cosh \left[\alpha_{1} h_{1}\right]+\beta \alpha_{1} \sinh \left[\alpha_{1} h_{1}\right]+1\right]$,

$a_{7}=\left[\sinh \left[\alpha_{1} h_{1}\right]+\beta \alpha_{1} \cosh \left[\alpha_{1} h_{1}\right]\right]$,

$B=\left[\frac{-(p+A)}{\frac{M^{2}}{1+m^{2}}+\frac{1}{D a}}\right], A=\frac{M^{2}}{1+m^{2}}+\frac{1}{D a}-\eta \sin \alpha$,

$\alpha_{1}=\sqrt{\left(\frac{M^{2}}{1+m^{2}}+\frac{1}{D a}\right)}$.

$\theta=A_{9} \cos [N y]+A_{8} \sin [N y]-\frac{v P_{r}}{N^{2}}-\left[\frac{M^{2} B_{r} A_{3}}{4 \alpha_{1}{ }^{2}+N^{2}}\right] e^{2 \alpha_{1} y}$

$-\left[\frac{M^{2} B_{r} A_{4}}{4 \alpha_{1}^{2}+N^{2}}\right] e^{-2 \alpha_{1} y}-\left[\frac{M^{2} B_{r} A_{5}}{\alpha_{1}^{2}+N^{2}}\right] e^{\alpha_{1} y}-\left[\frac{M^{2} B_{r} A_{6}}{\alpha_{1}^{2}+N^{2}}\right] e^{-\alpha_{1} y}$

$-\left[\frac{M^{2} B_{r} A_{7}}{N^{2}}\right]$

where

$$
\begin{aligned}
& A_{3}=\left[\frac{A_{1}^{2}}{4}+\frac{{A_{2}}^{2}}{4}+\frac{A_{1} A_{2}}{2}\right], A_{4}=\left[\frac{{A_{1}}^{2}}{4}+\frac{{A_{2}}^{2}}{4}-\frac{A_{1} A_{2}}{2}\right], \\
& A_{5}=\left[B\left(A_{1}+A_{2}\right)\right], A_{6}=\left[B\left(A_{2}-A\right)\right] \text {, } \\
& A_{7}=\left[-\frac{A_{1}^{2}}{2}+\frac{A_{2}^{2}}{2}+B^{2}\right] \text {, } \\
& A_{8}= \\
& {\left[\frac{1}{a_{14}}\right]\left[-\cos \left[N h_{1}\right]-a_{8}-a_{9}-a_{10}-a_{11}-a_{12}-a_{13}\right],} \\
& A_{9}=\left[\frac{1}{\cos \left[N h_{1}\right]}\right]\left[-A_{8} \sin \left[N h_{1}\right]+\frac{v P_{r}}{N^{2}}+\left[\frac{M^{2} B_{r} A_{3}}{4 \alpha_{1}{ }^{2}+N^{2}}\right] e^{2 \alpha_{1} h_{1}}\right. \\
& +\left[\frac{M^{2} B_{r} A_{4}}{4 \alpha_{1}^{2}+N^{2}}\right] e^{-2 \alpha_{1} h_{1}}+\left[\frac{M^{2} B_{r} A_{5}}{\alpha_{1}^{2}+N^{2}}\right] e^{\alpha_{1} h_{1}}+\left[\frac{M^{2} B_{r} A_{6}}{\alpha_{1}^{2}+N^{2}}\right] e^{-\alpha_{1} h_{1}} \\
& \left.+\frac{M^{2} B_{r} A_{7}}{N^{2}}\right] \\
& a_{8}=\left[\left(\frac{v P_{r}}{N^{2}}\right)\left(\cos \left[N h_{1}\right]-\cos \left[N h_{2}\right]\right)\right] \text {, } \\
& a_{9}=\left[\left(\frac{M^{2} B_{r} A_{3}}{4 \alpha_{1}^{2}+N^{2}}\right)\left(e^{2 \alpha_{1} h_{2}} \cos \left[N h_{1}\right]-e^{2 \alpha_{1} h_{2}} \cos \left[N h_{2}\right]\right)\right] \text {, } \\
& a_{10}=\left[\left(\frac{M^{2} B_{r} a_{4}}{4 \alpha_{1}{ }^{2}+N^{2}}\right)\left(e^{-2 \alpha_{1} h_{2}} \cos \left[N h_{1}\right]-e^{-2 \alpha_{1} h_{2}} \cos \left[N h_{2}\right]\right)\right], \\
& a_{11}=\left[\left(\frac{M^{2} B_{r} A_{5}}{\alpha_{1}^{2}+N^{2}}\right)\left(e^{\alpha_{1} h_{2}} \cos \left[N h_{1}\right]-e^{\alpha_{1} h_{2}} \cosh \left[N h_{2}\right]\right)\right] \text {, } \\
& a_{12}=\left[\left(\frac{M^{2} B_{r} A_{6}}{\alpha_{1}^{2}+N^{2}}\right)\left(e^{-\alpha_{1} h_{2}} \cos \left[N h_{1}\right]-e^{-\alpha_{1} h_{1}} \cosh \left[N h_{2}\right]\right)\right] \text {, } \\
& a_{13}=\left[\left(\frac{M^{2} B_{r} A_{7}}{N^{2}}\right)\left(\cos \left[N h_{1}\right]-\cos \left[N h_{1}\right]\right)\right] \text {, } \\
& a_{14}=\left[\sinh \left[N h_{1}\right] \cos \left[N h_{2}\right]-\sinh \left[N h_{2}\right] \cos \left[N h_{1}\right]\right] \text {. } \\
& \Phi=A_{24} \sinh [d y]+A_{23} \cos [\mathrm{dy}]+A_{16} \cos [\mathrm{Ny}] \\
& +A_{17} \sin [\mathrm{Ny}]+A_{18} e^{2 \alpha_{1} y}+A_{19} e^{-2 \alpha_{1} y} \\
& +A_{20} e^{\alpha_{1} y}+A_{21} e^{-\alpha_{1} y} \\
& A_{10}=A_{9} N^{2} S_{c} S_{r}, \quad A_{11}=A_{8} N^{2} S_{c} S_{r} \text {, } \\
& A_{12}=\frac{4 A_{3} \alpha_{1}^{2} M^{2} B_{r} S_{C} S_{r}}{4 \alpha_{1}^{2}+N^{2}}, A_{13}=\frac{4 A_{4} \alpha_{1}^{2} M^{2} B_{r} S_{C} S_{r}}{4 \alpha_{1}^{2}+N^{2}}, \\
& A_{15}=\frac{A_{6} \alpha_{1}{ }^{2} M^{2} B_{r} S_{C} S_{r}}{\alpha_{1}{ }^{2}+N^{2}}, A_{14}=\frac{A_{5} \alpha_{1}{ }^{2} M^{2} B_{r} S_{C} S_{r}}{\alpha_{1}{ }^{2}+N^{2}}, A_{16}=\left(\frac{-A_{10}}{n^{2}+d^{2}}\right) \text {, } \\
& A_{17}=\left(\frac{-A_{11}}{n^{2}+d^{2}}\right), \quad A_{18}=\left(\frac{A_{12}}{4 \alpha_{1}^{2}-d^{2}}\right), A_{19}=\left(\frac{A_{13}}{4 \alpha_{1}^{2}-d^{2}}\right), \\
& A_{20}=\left(\frac{A_{14}}{\alpha_{1}^{2}-d^{2}}\right), A_{21}=\left(\frac{A_{15}}{\alpha_{1}^{2}-d^{2}}\right) \text {, } \\
& A_{22}=A_{16}\left(\cos \left[\mathrm{N} h_{1}\right] \sinh \left[\mathrm{d} h_{2}\right]-\cos \left[\mathrm{N} h_{2}\right] \sinh \left[\mathrm{d} h_{1}\right]\right) \\
& +A_{17}\left(\sin \left[\mathrm{N} h_{1}\right] \sinh \left[\mathrm{d} h_{2}\right]-\sin \left[\mathrm{N} h_{1}\right] \sinh \left[\mathrm{d} h_{2}\right] \sinh \left[\mathrm{d} h_{1}\right]\right)
\end{aligned}
$$




$$
\begin{aligned}
& +A_{18}\left(\sinh \left[\mathrm{d} h_{2}\right] e^{2 \alpha_{1} h_{1}}-\sinh \left[\mathrm{d} h_{1}\right] e^{2 \alpha_{1} h_{2}}\right) \\
& +A_{19}\left(\sinh \left[\mathrm{d} h_{2}\right] e^{-2 \alpha_{1} h_{1}}-\sinh \left[\mathrm{d} h_{1}\right] e^{2 \alpha_{1} h_{2}}\right) \\
& +A_{20}\left(\sinh \left[\mathrm{d} h_{2}\right] e^{\alpha_{1} h_{1}}-\sinh \left[\mathrm{d} h_{1}\right] e^{\alpha_{1} h_{2}}\right) \\
& +A_{21}\left(\sinh \left[\mathrm{d} h_{2}\right] e^{-\alpha_{1} h_{1}}-\sinh \left[\mathrm{d} h_{1}\right] e^{-\alpha_{1} h_{2}}\right), \\
A_{23}= & {\left[\frac{\sinh \left[\mathrm{d} h_{1}\right]-A_{22}}{\left[\sinh \left[d h_{2}\right] \cosh \left[d h_{1}\right]-\sinh \left[d h_{1}\right] \cosh \left[d h_{2}\right]\right]}\right] } \\
A_{24}= & \left(\frac{1}{\sinh \left[\mathrm{d} h_{1}\right]}\right)\left(-A_{23} \cosh \left[\mathrm{d} h_{1}\right]-A_{16} \cos \left[\mathrm{N} h_{1}\right]\right) \\
& +\left(\frac{1}{\sinh \left[\mathrm{d} h_{1}\right]}\right)\left(-A_{17} \sin \left[\mathrm{N} h_{1}\right]-A_{18} e^{2 \alpha_{1} h_{1}}\right) \\
& +\left(\frac{1}{\sinh \left[\mathrm{d} h_{1}\right]}\right)\left(-A_{19} e^{-2 \alpha_{1} h_{1}}-A_{20} e^{\alpha_{1} h_{1}}-A_{21} e^{-\alpha_{1} h_{1}}\right) .
\end{aligned}
$$

Heat transfer rate and mass transfer rate at $\mathrm{y}=\mathrm{h}_{1}$ wall are defined in terms of Nusselt number $(\mathrm{Nu})$ and Sherwood number (Sh) as follows

$$
\begin{aligned}
N u= & \theta^{I}\left(h_{1}\right)\left(h_{1}\right)_{x}=\left(-N A_{9} \sin \left[N h_{1}\right]+A_{8} N \cos \left[N h_{1}\right]\right. \\
& -\left[\frac{2 \alpha_{1} M^{2} B_{r} A_{3}}{4 \alpha_{1}^{2}+N^{2}}\right] e^{2 \alpha_{1} h_{1}}-\left[\frac{-2 \alpha_{1} M^{2} B_{r} A_{4}}{4 \alpha_{1}^{2}+N^{2}}\right] e^{-2 \alpha_{1} h_{1}} \\
& \left.-\left[\frac{\alpha_{1} M^{2} B_{r} A_{5}}{\alpha_{1}^{2}+N^{2}}\right] e^{\alpha_{1} h_{1}}-\left[\frac{-\alpha_{1} M^{2} B_{r} A_{6}}{\alpha_{1}^{2}+N^{2}}\right] e^{-\alpha_{1} h_{1}}\right) \\
& \times\left(-2 \pi \cos [2 \pi(x-t)+\emptyset]-k_{1}\right)
\end{aligned}
$$

$$
\begin{aligned}
S h= & \Phi^{I}\left(h_{1}\right)\left(h_{1}\right)_{x}=\left(A_{24} d \cosh \left[d h_{1}\right]+A_{23} \sinh \left[d h_{1}\right]\right. \\
& -A_{16} \mathrm{~N} \sin \left[\mathrm{N} h_{1}\right]+A_{17} N \cos \left[N h_{1}\right]+A_{18} 2 \alpha_{1} e^{2 \alpha_{1} h_{1}} \\
& \left.-A_{19} 2 \alpha_{1} e^{-2 \alpha_{1} h_{1}}+A_{20} \alpha_{1} e^{\alpha_{1} h_{1}}-A_{21} \alpha_{1} e^{-\alpha_{1} h_{1}}\right) \\
& \times\left(-2 \pi \cos [2 \pi(x-t)+\emptyset]-k_{1}\right)
\end{aligned}
$$

The volumetric flow rate in the wave frame is defined by

$$
\begin{aligned}
q & =\int_{h_{1}}^{h_{2}} u d y=\int_{h_{1}}^{h_{2}}\left(A_{1} \sinh \left[\alpha_{1} y\right]+A_{2} \cosh \left[\alpha_{1} y\right]+B\right) d y \\
& =B a_{4}
\end{aligned}
$$

$$
\begin{aligned}
a_{4}= & {\left[-\frac{a_{3}}{\alpha_{1}}\left[\cosh \left[\alpha_{1} h_{2}\right]-\cosh \left[\alpha_{1} h_{1}\right]\right]\right.} \\
& \left.+\frac{a_{2}}{\alpha_{1}}\left[\sinh \left[\alpha_{1} h_{2}\right]-\sinh \left[\alpha_{1} h_{1}\right]\right]+\left[\left(h_{2}-h_{1}\right)\right]\right]
\end{aligned}
$$

The pressure gradient obtained from equation (27) can be expressed as

$\frac{d p}{d x}=-\left[\frac{q}{a_{4}}\left[\frac{M^{2}}{1+m^{2}}+\frac{1}{D}\right]+A\right]$

The instantaneous flux $\mathrm{Q}(\mathrm{x}, \mathrm{t})$ in the laboratory frame is

$Q=\int_{h_{2}}^{h_{1}}(u+1) d y=q-h$

where

$$
\begin{aligned}
\mathrm{q}= & B a_{4}, \\
a_{4}= & {\left[-\frac{a_{3}}{\alpha_{1}}\left[\cosh \left[\alpha_{1} h_{2}\right]-\cosh \left[\alpha_{1} h_{1}\right]\right]\right.} \\
& \left.+\frac{a_{2}}{\alpha_{1}}\left[\sinh \left[\alpha_{1} h_{2}\right]-\sinh \left[\alpha_{1} h_{1}\right]\right]+\left[\left(h_{2}-h_{1}\right)\right]\right] .
\end{aligned}
$$

The average volume flow rate over one wave period $(\mathrm{T}=$ $\lambda / \mathrm{c}$ ) of the peristaltic wave is defined as

$\bar{Q}=\frac{1}{T} \int_{0}^{T} Q d t=q+1+d$

where

$$
\begin{aligned}
\mathrm{q}= & B a_{4}, \\
a_{4}= & {\left[-\frac{a_{3}}{\alpha_{1}}\left[\cosh \left[\alpha_{1} h_{2}\right]-\cosh \left[\alpha_{1} h_{1}\right]\right]\right.} \\
& \left.+\frac{a_{2}}{\alpha_{1}}\left[\sinh \left[\alpha_{1} h_{2}\right]-\sinh \left[\alpha_{1} h_{1}\right]\right]+\left[\left(h_{2}-h_{1}\right)\right]\right] .
\end{aligned}
$$

From the equations (28) and (30), the pressure gradient can be expressed as

$\frac{d p}{d x}=-\left[\frac{(\bar{Q}-1-d)}{a_{4}}\left[\frac{M^{2}}{1+m^{2}}+\frac{1}{D}\right]+A\right]$

\section{NUMERICAL RESULTS AND DISCUSSION}

The objective of this research is to study hall current, radiation and velocity slip on hydromagnetic physiological hemodynamic fluid with joule heating and mass transfer in presence of chemical reaction in an inclined asymmetrical tapered vertical channel.

The influence of hartmann number $(\mathrm{M})$, gravitational parameter $(\eta)$, slip parameter $(\beta)$, porosity parameter $(\mathrm{Da})$, hall parameter $(\mathrm{m})$ and volumetrical rate of flow $(\bar{Q})$ on pressure gradient are shown graphically in fig. 2. The following default parameter values are adopted for computations: $\mathrm{M}=2, \mathrm{Da}=0.1, \beta=0.7, \overline{\mathrm{Q}}=0.2, \mathrm{~m}=0.5, \mathrm{t}=$ $\frac{\pi}{4}, \mathrm{k}_{1}=0.1, \mathrm{~d}=2, \eta=0.5, \phi=\pi / 6, \alpha=\pi / 6, \varepsilon=0.2$.

Fig. 2(a) is plotted for various values of hartmann number $(\mathrm{M}=1,1.5,2)$ on axial pressure gradient. It shows that increases in $\mathrm{M}$, the fluid in pressure gradient rises throughout the channel. Figure 2(b) indicates the effect of the gravitational parameter $(\eta)$ on $\frac{d p}{d x}$ with fixed other parameters. It is realized that the results in pressure gradient increase in the entire channel by increasing gravitational parameter $(\eta=$ $0.1,0.5,1)$. In figure $2(\mathrm{c})$, we analyzed that for different values of slip parameter $(\beta=0.7,0.8,0.9)$, the pressure gradient $\left(\frac{d p}{d x}\right)$ enhances. Therefore, we conclude from these graphs $(2(a)-2(c))$ that it requires large pressure gradient to maintain same flux to pass in the asymmetric tapered inclined vertical channel. Figures $2(d)-2(f)$ focus on the on pressure gradient $\left(\frac{d p}{d x}\right)$ for different values of porosity parameter $(\mathrm{Da})$, hall current parameter $(\mathrm{m})$ and volumetrical rate of flow $(\overline{\mathrm{Q}})$ respectively. We perceive from these graphs that the fluid of the pressure gradient reduces throughout the channel by an increase in porosity parameter ( $\mathrm{Da} 0.1,0.2,0.3$ ), hall current parameter $(\mathrm{m}=1,1.5,2)$ and volumetrical rate of flow $(\overline{\mathrm{Q}}=$ $0.1,0.3,0.5)$.

The effect of hartmann number $(\mathrm{M})$, porosity parameter $(\mathrm{Da})$, radiation parameter $(\mathrm{N})$, prandtl number $(\mathrm{Pr})$, brinkman number $(\mathrm{Br})$, heat source parameter $(v)$, slip parameter $(\beta)$ and hall parameter $(\mathrm{m})$ on temperature distribution are shown graphically in fig. 3 . The following default parameter values are adopted for computations: $\mathrm{M}=2, \mathrm{~N}=0.5, \mathrm{Pr}=5, \mathrm{~m}=1$, $\eta=0.5, \beta=0.7, \beta=0.5, \mathrm{Da}=0.1, \mathrm{Br}=0.3, v=0.1, \phi=\pi / 6$, $\alpha=\pi / 6, \mathrm{k}_{1}=0.1, \mathrm{p}=-1, \mathrm{t}=0.4, \mathrm{x}=0.6, \varepsilon=0.2$. Figure 3(a) reveals that the effect of hartmann number on temperature distribution. It is clear that the temperature of the fluid is always positive and it enhances by an increase in hartmann number $(\mathrm{M}=1,1.5,2)$. This enhancement in temperature due to consideration of joule heating. Fig. 3(b) depicts to examine the effect porosity parameter on temperature distribution. This graph indicates that temperature increases with an 
increase of porosity parameter $(\mathrm{Da}=0.1,0.2,0.3)$. But the enhancement in the temperature distribution is not significant by an increase in porosity parameter. 3(c) presents the various values of radiation parameter $(\mathrm{N}=0.3,0.5,0.7)$ on temperature profile $(\theta)$. We perceive from this graph that the increase in radiation parameter, the results in temperature profile increases. Effect of prandtl number (Pr) on the temperature of the fluid $(\theta)$ is presented in fig 3(d). It is clear that the temperature of the fluid enhances with an increase in prandtl number $(\operatorname{Pr}=5,7,9)$. Figure 3(e) analyzes the impact of brinkman number $(\mathrm{Br})$ on the temperature distribution. It observed that the temperature increases when the increase in brinkman number $(\mathrm{Br}=0.1,0.2,0.3)$. Fig.3 (f) elucidates that the influence of heat source parameter on temperature distribution. Indeed, the temperature of the fluid increases significantly with increase in heat source parameter $(v=0.1$, $0.3,0.5)$. The relation between temperature and slip condition parameter is presented in figure $3(\mathrm{~g})$. It is interesting to note that the temperature of the fluid reduces when an increase in slip parameter $(\beta=0.7,0.8,0.9)$. This deterioration in temperature subject to increase in $\beta$ and also we notice that the fluid of the temperature is not significant. Influence of various values of hall parameter $(\mathrm{m}=1,1.5,2)$ on temperature distribution is presented in figure 3(h) with fixed other parameters. This graph reveals the temperature distribution increases by the increase in $\mathrm{m}$.

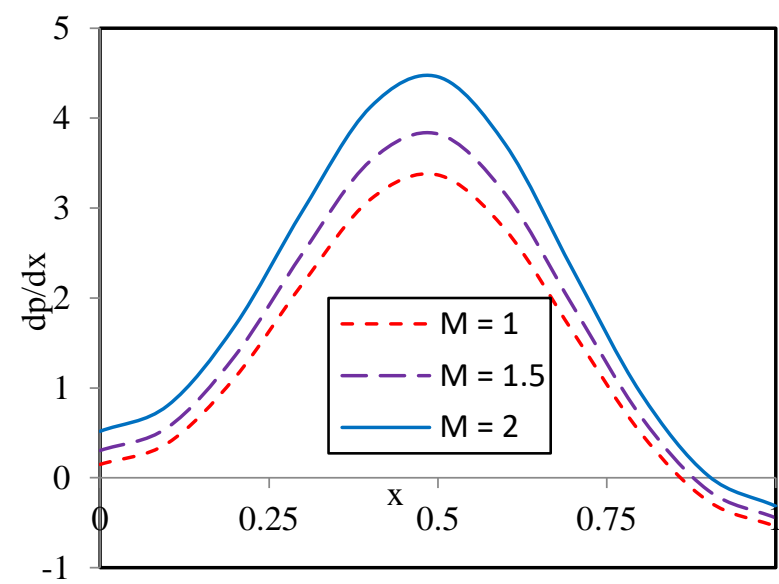

Figure 2 (a). Impact of $\mathrm{M}$ on Pressure gradient $\left(\frac{d p}{d x}\right)$

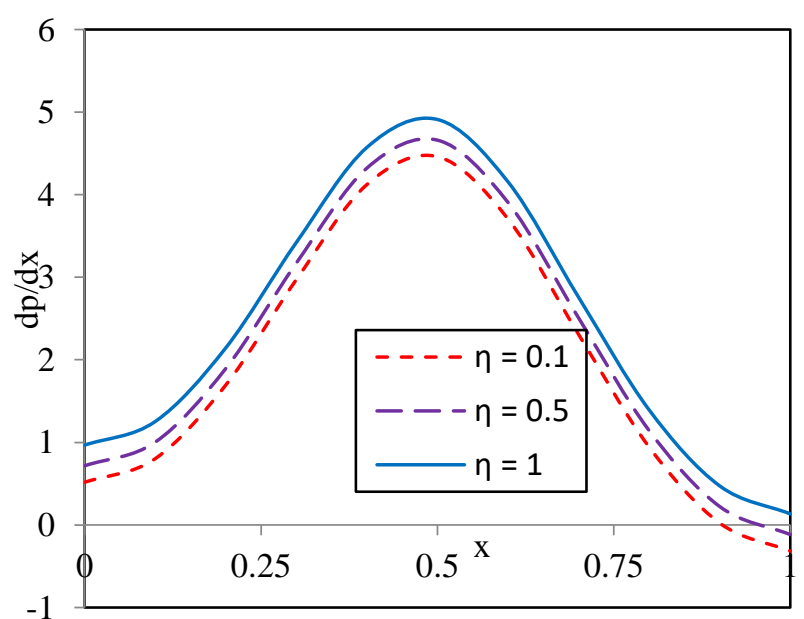

Figure 2(b). Impact of $\eta$ on Pressure gradient $\left(\frac{d p}{d x}\right)$

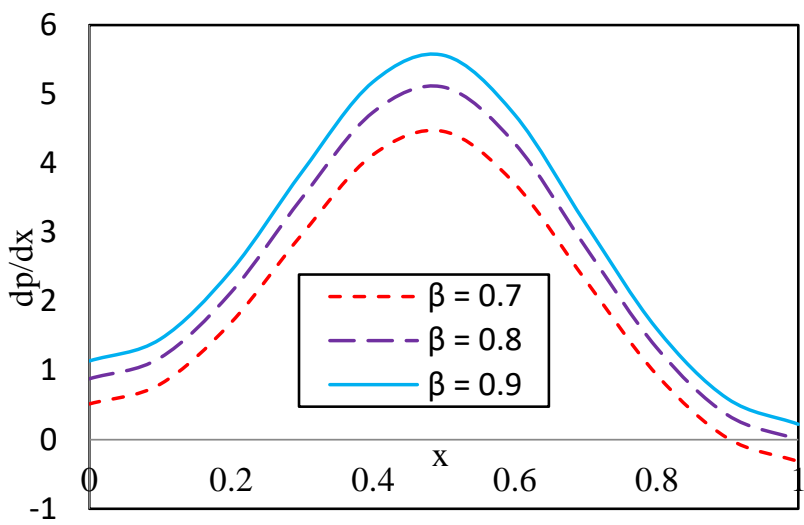

Figure 2(c). Impact of $\beta$ on Pressure gradient $\left(\frac{d p}{d x}\right)$

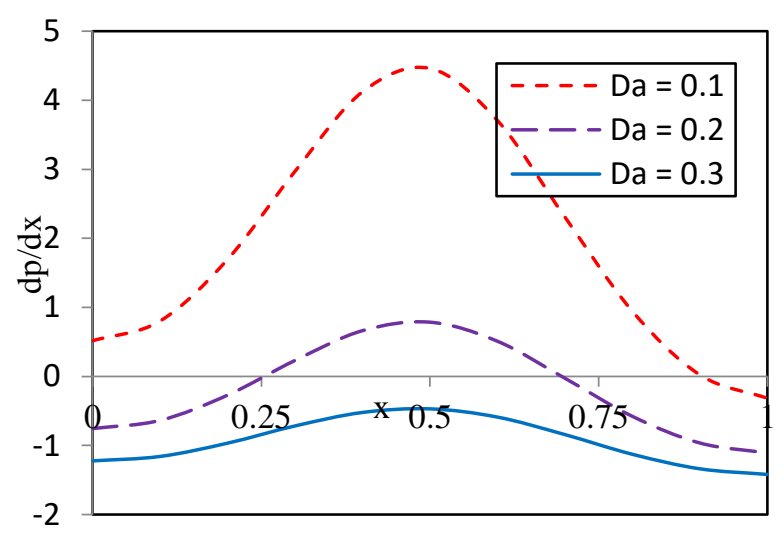

Figure 2(d). Impact of Da on Pressure gradient $\left(\frac{d p}{d x}\right)$

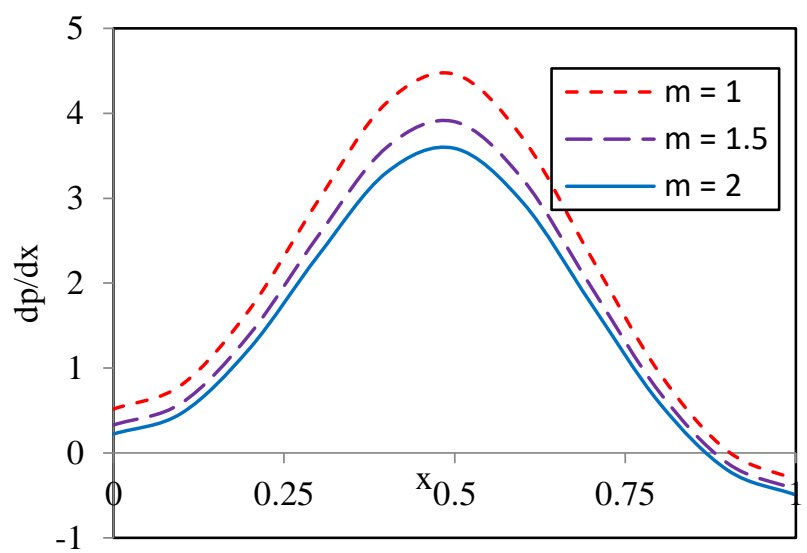

Figure 2 (e). Impact of $\mathrm{m}$ on Pressure gradient $\left(\frac{d p}{d x}\right)$

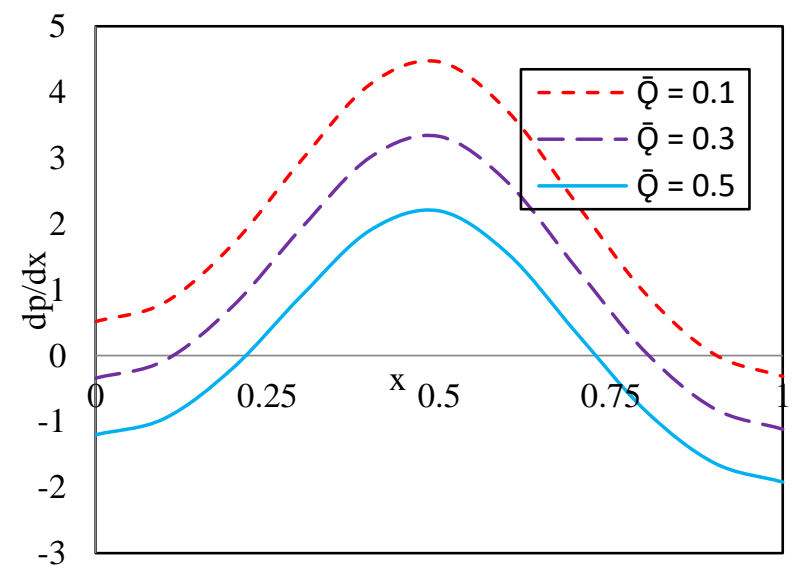

Figure 2(f). Impact of $\bar{Q}$ on Pressure gradient $\left(\frac{d p}{d x}\right)$ 


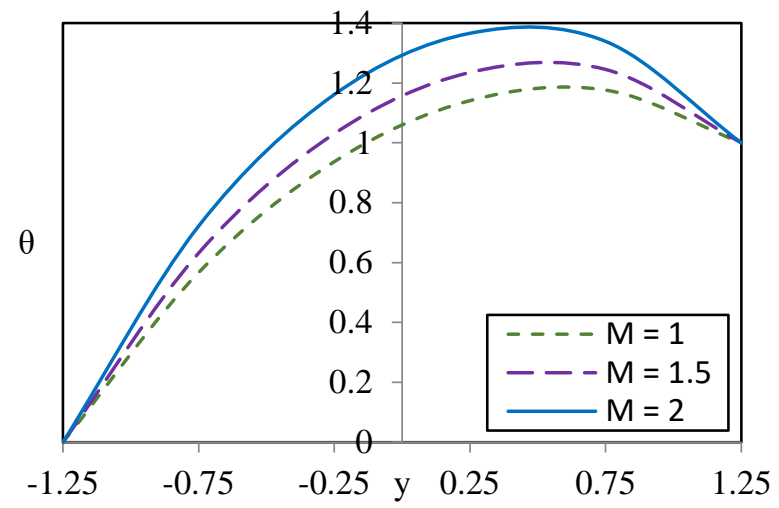

Figure 3(a). Impact of $\mathrm{M}$ on Temperature distribution $(\theta)$

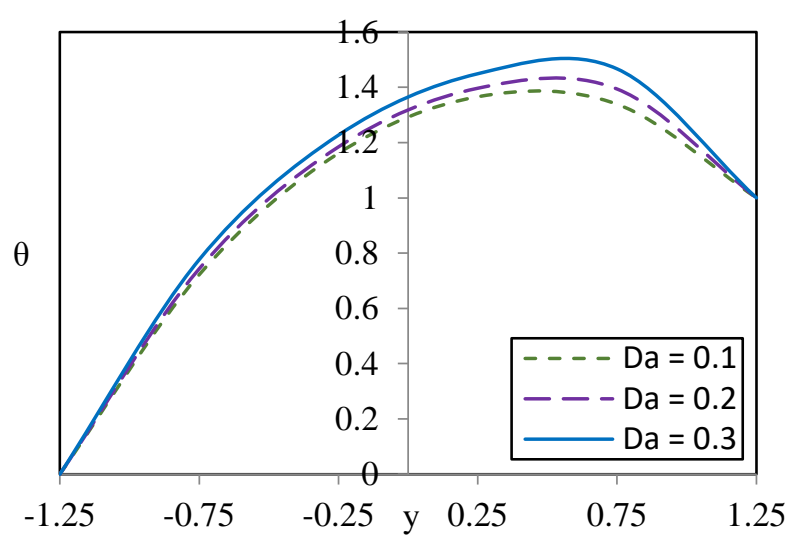

Figure 3(b). Impact of Da on Temperature distribution $(\theta)$

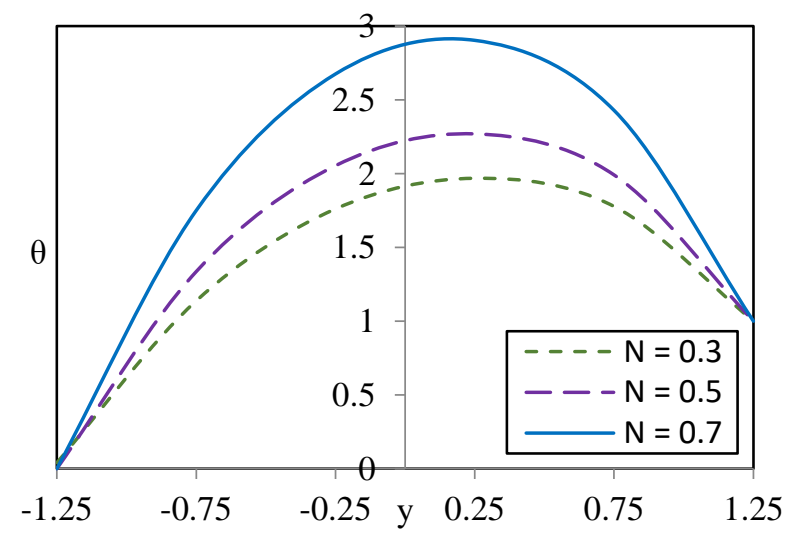

Figure 3(c). Impact of $\mathrm{N}$ on Temperature distribution $(\theta)$

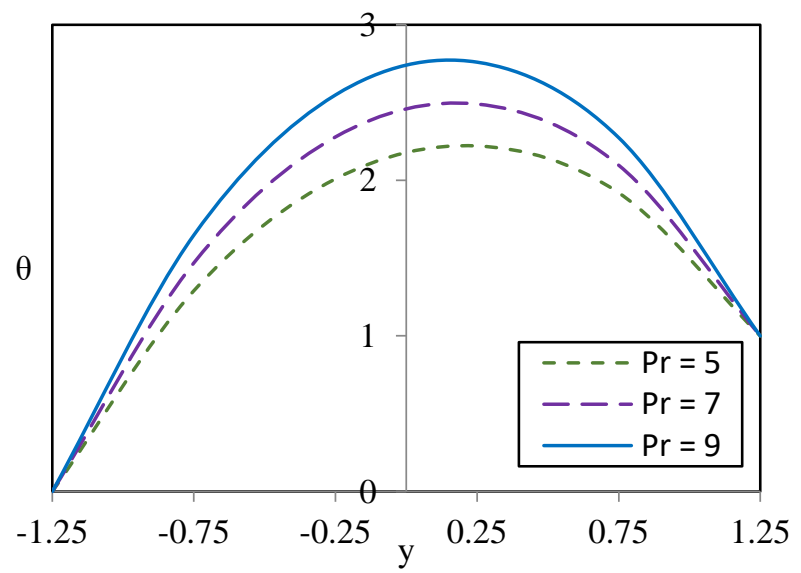

Figure 3(d). Impact of Pr on Temperature distribution $(\theta)$

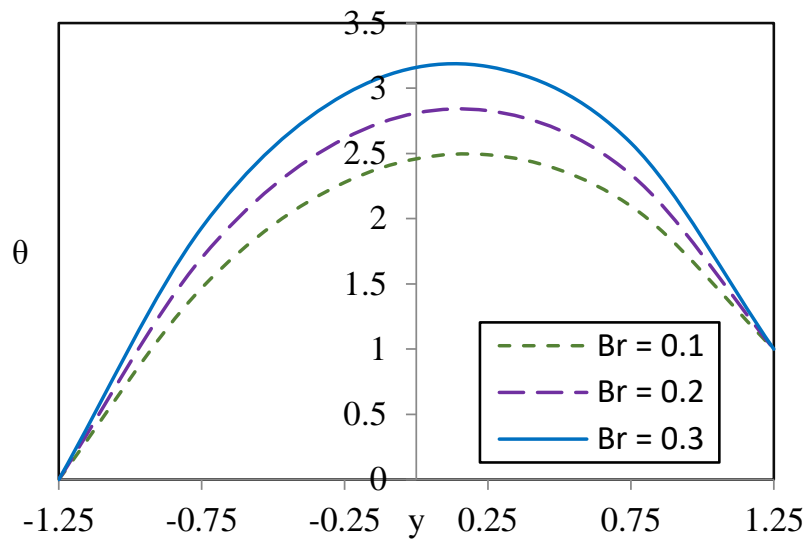

Figure 3(e). Impact of $\mathrm{Br}$ on Temperature distribution $(\theta)$

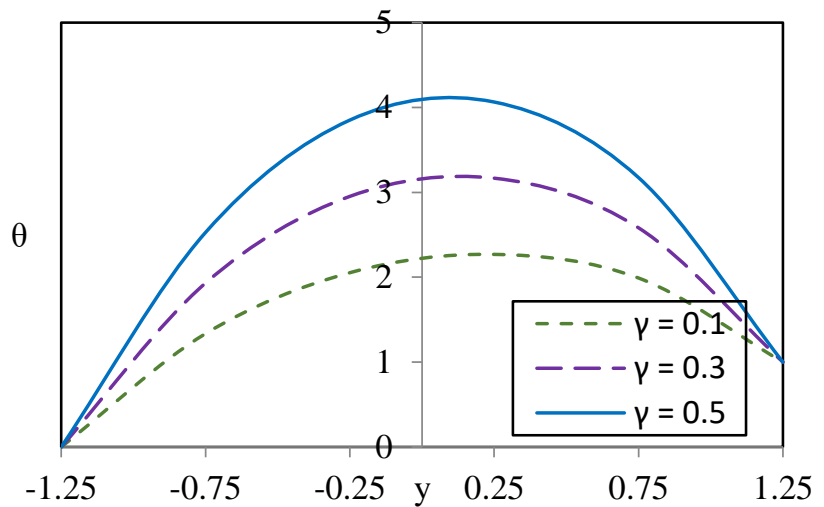

Figure 3(f). Impact of $v$ on Temperature distribution $(\theta)$

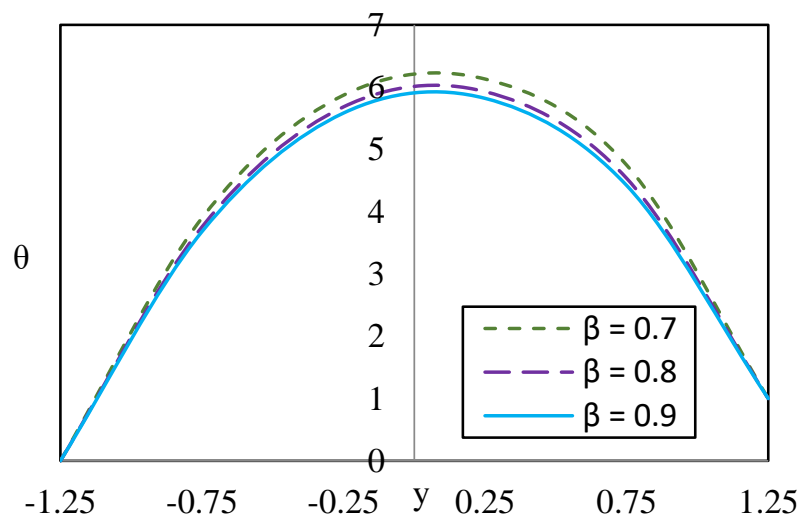

Figure 3(g). Impact of $\beta$ on Temperature distribution $(\theta)$

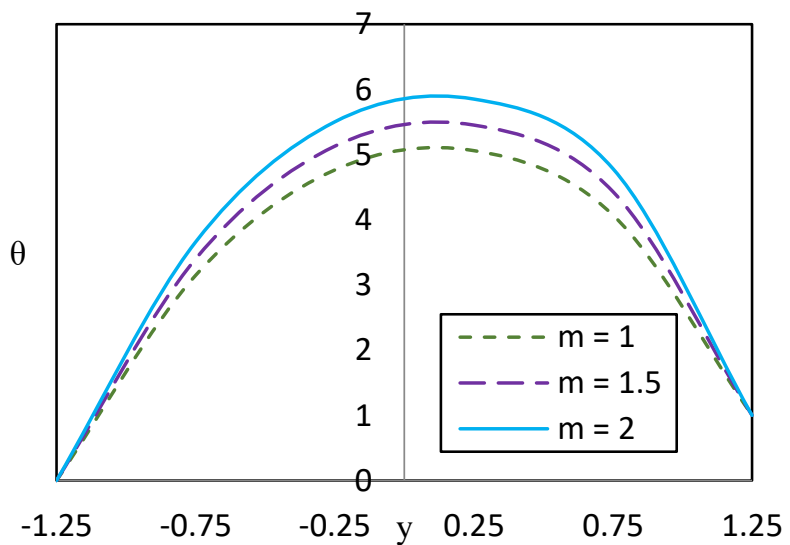

Figure 3(h). Impact of $\mathrm{m}$ on Temperature distribution $(\theta)$ 
The influence of hartmann number (M), porosity parameter $(\mathrm{Da})$, radiation parameter $(\mathrm{N})$, prandtl number $(\mathrm{Pr})$, brinkman number $(\mathrm{Br})$, heat source parameter(v), hall parameter $(\mathrm{m})$, chemical reaction $(\mathrm{S})$, schmidt number $(\mathrm{Sc})$ and soret number $(\mathrm{Sr})$ on concentration distribution $(\Phi)$ are shown graphically in fig. 4 . The following default parameter values are adopted for computations: $\mathrm{Da}=0.1, \mathrm{~N}=0.3, \mathrm{Pr}=$ $5, \mathrm{~m}=1, \mathrm{Sc}=0.3, \mathrm{Sr}=3, \mathrm{~S}=1.5 \eta=0.5, \beta=0.7, \mathrm{Br}=0.3, v$ $=0.1, \phi=\pi / 6, \alpha=\pi / 6, \mathrm{k}_{1}=0.1, \mathrm{p}=-1, \mathrm{t}=0.4, \mathrm{x}=0.6, \varepsilon=$ 0.2 .

Fig. 4(a) illustrates the effect of hartmann number on the concentration profile $(\Phi)$ of the flow. It is clear that an increase in hartmann number $(\mathrm{M}=1,1.5,2)$ declines the concentration profile of the flow. The relation between concentration distribution $(\Phi)$ and porosity parameter has been presented in fig.4(b). We perceive from this graph that the fluid of concentration reduces with an increase in porosity parameter $(\mathrm{Da}=0.1,0.2,0.3)$. The impact of radiation parameter on concentration profile is shown in figure 4(c). We notice from this graph that the concentration distribution decreases with an increase in radiation parameter $(\mathrm{N}=0.3$, $0.5,0.7)$. Fig. 4(d) displays the variation of prandtl number on concentration distribution. It can be seen that an increase in prandtl number $\operatorname{Pr}$ causes a decrease in concentration because a rise Pr enhances the viscosity of the fluid which turns decline the concentration of the fluid. Effects of Brinkman number $(\mathrm{Br})$ and Heat source parameter $(v)$ on concentration distribution are plotted in figures 4(e) and 4(f). We observed that the concentration reduces when brinkman number $(\mathrm{Br})$ and heat source parameter $(v)$ increased. Figures 4(g)-4(i) reveal the concentration distribution with various values of hall current parameter $(\mathrm{m})$, schmidt number $(\mathrm{Sc})$ and soret number $(\mathrm{Sr})$. It was evident through these graphs that the fluid in concentration distribution diminishes with an increase in hall current parameter $(\mathrm{m}=1,1.5,2)$, schmidt number $(\mathrm{Sc}=0.3,0.5,0.7)$ and soret number $(\mathrm{Sr}=1,3,5)$. An important result presented in fig. 4(j). It is interesting to note that an increase in chemical reaction parameter causes an increase in the concentration of the fluid.

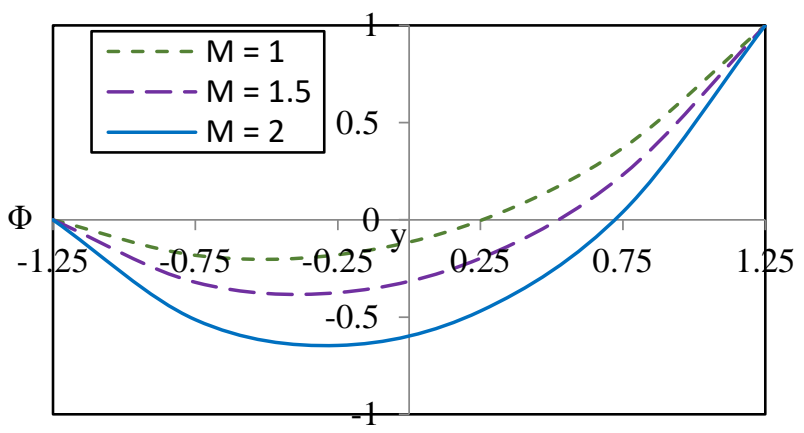

Figure 4(a). Impact of $M$ on $\Phi$

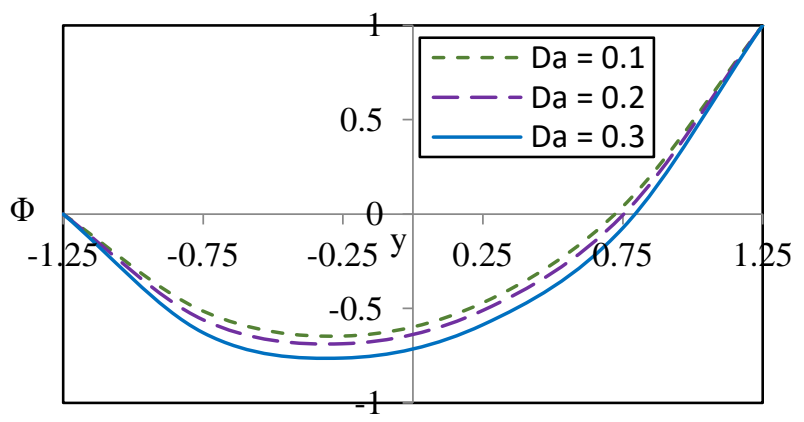

Figure 4(b). Impact of Da on $\Phi$

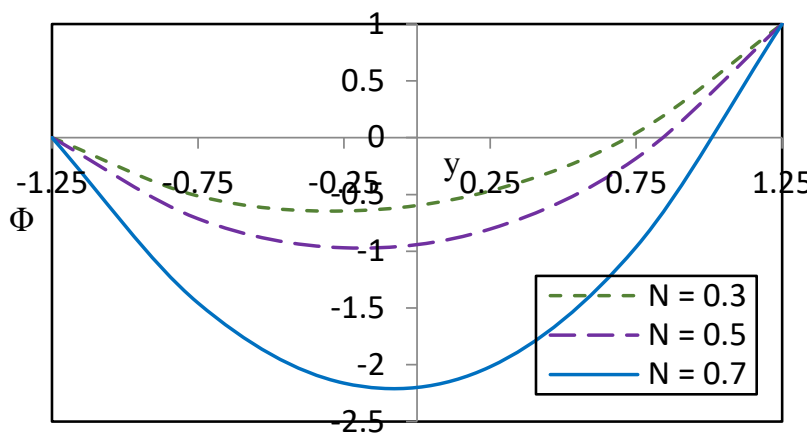

Figure 4(c). Impact of $N$ on $\Phi$

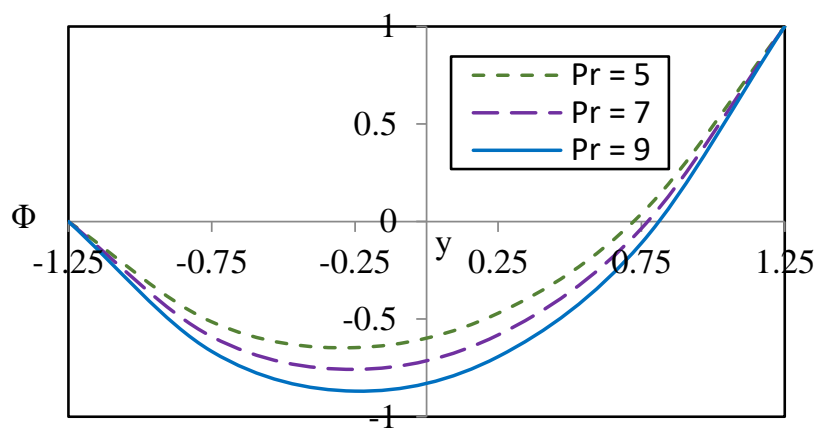

Figure 4(d). Impact of $\operatorname{Pr}$ on $\Phi$

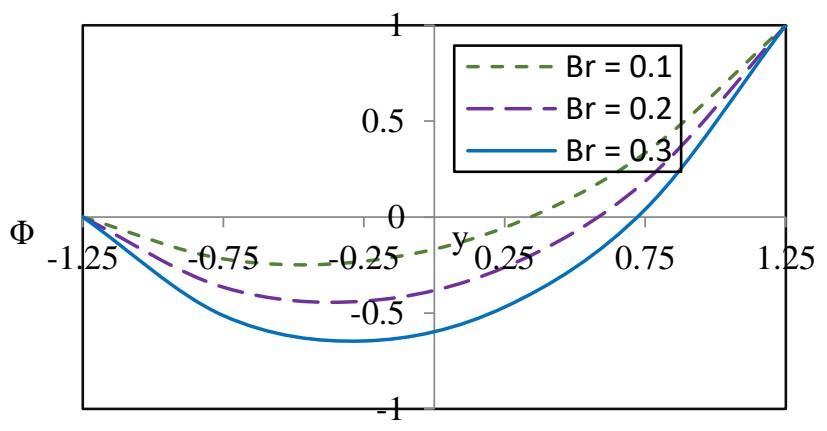

Figure 4(e). Impact of $\mathrm{Br}$ on $\Phi$

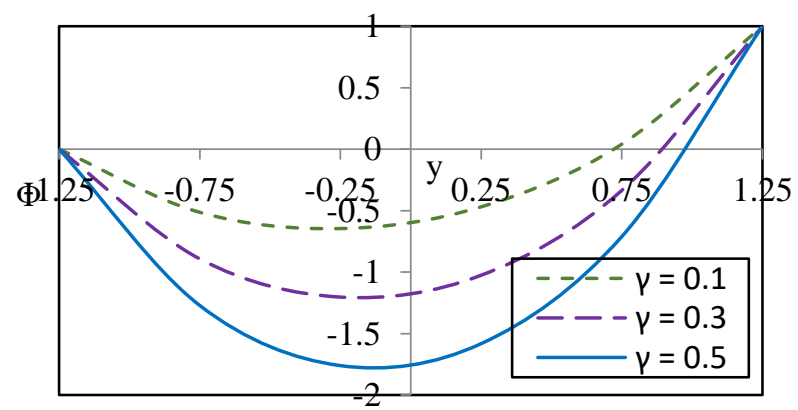

Figure 4(f). Impact of $v$ on $\Phi$

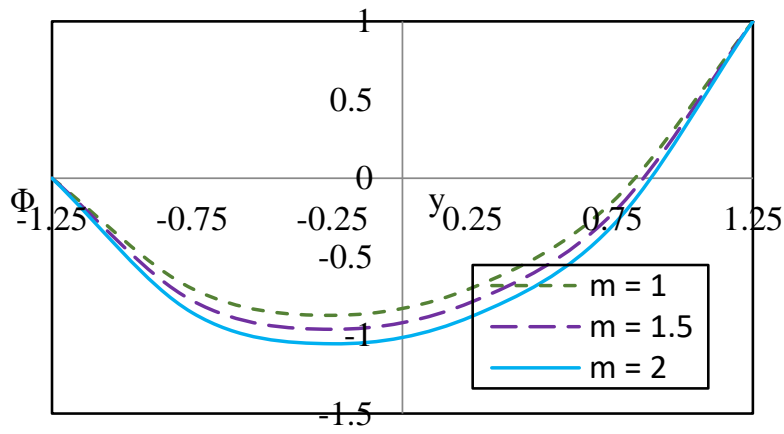

Figure 4(g). Impact of $m$ on $\Phi$ 


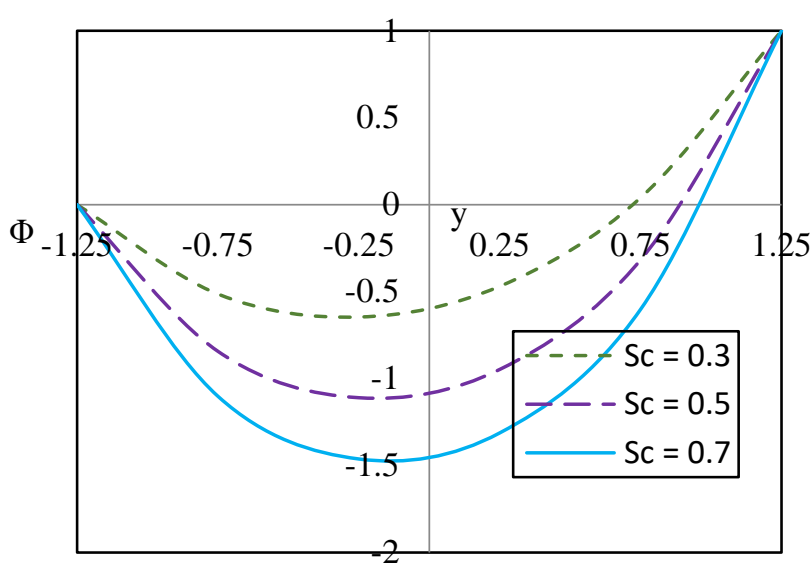

Figure 4(h). Impact of Sc on $\Phi$

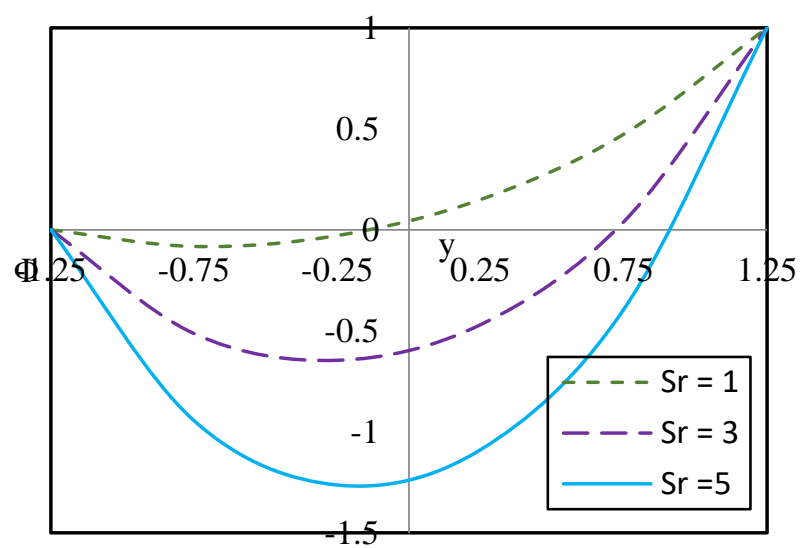

Figure 4(i). Impact of $\mathrm{Sr}$ on $\Phi$

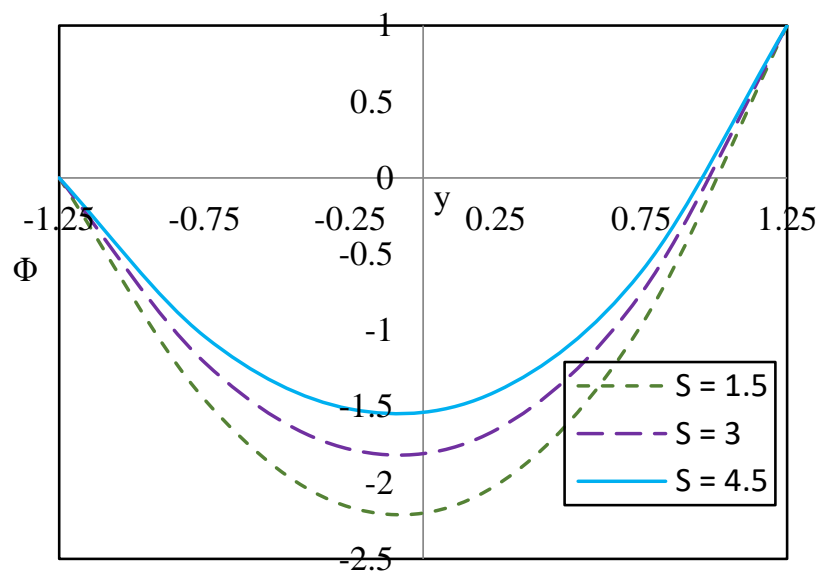

Figure 4(j). Impact of $S$ on $\Phi$

The variations of nusselt number $(\mathrm{Nu})$ at the wall $\mathrm{y}=\mathrm{h}_{1}$ for various values of physical parameters are plotted in figure 5. The following default parameter values are adopted for computations: $\mathrm{Da}=0.1, \mathrm{M}=2, \mathrm{~N}=0.3, \mathrm{~m}=1, \eta=0.5, \beta=$ $0.7, \beta=0.5, \mathrm{Br}=0.3, \operatorname{Pr}=5, v=0.1, \phi=\pi / 6, \alpha=\pi / 6, \mathrm{k}_{1}=$ $0.1, \mathrm{p}=-1, \mathrm{t}=0.4, \varepsilon=0.2$. Fig. 5(a) to 5(c) illustrates the effects of radiation parameter, brinkman number and hall current parameter on the variation of heat transfer rate (Nusselt number) $\mathrm{Nu}$. We observed from these graphs that the absolute value of nusselt number enhances in the region $\mathrm{x}$ $\in[0,0.05] \mathrm{U}[0.57,1]$ while it reduces in the rest of the region $\mathrm{x} \in[00.5,0.57]$ by increase in radiation parameter $(\mathrm{N}$ $=0.3,0.5,0.7)$, brinkman number $(\mathrm{Br}=0.1,0.2,0.3)$ and hall current parameter $(\mathrm{m}=1,1.5,2)$.

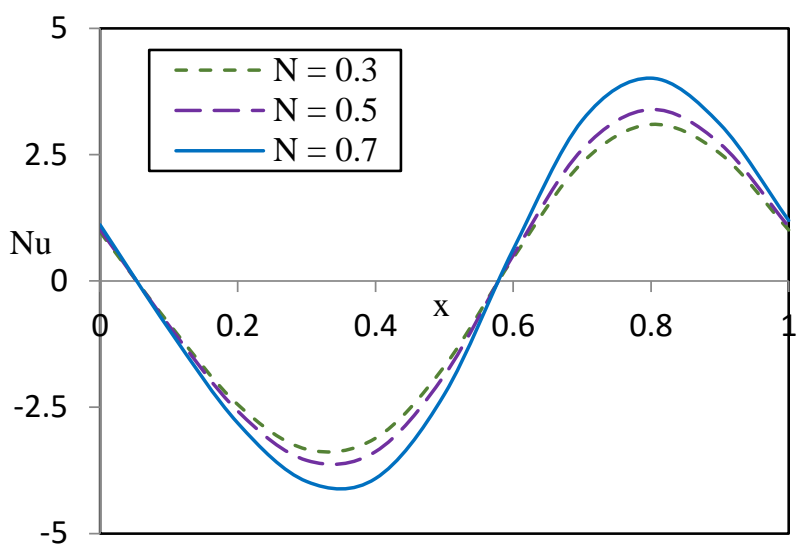

Figure 5(a). Impact of $\mathrm{N}$ on Nusselt number $(\mathrm{Nu})$

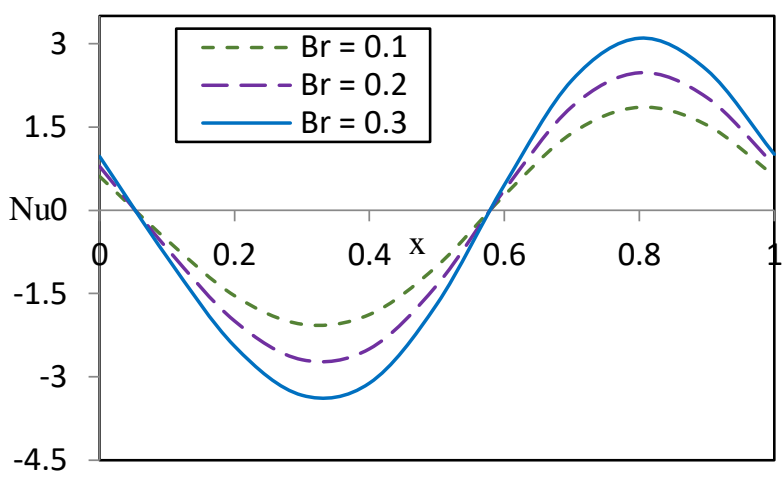

Figure 5(b). Impact of $\mathrm{Br}$ on Nusselt number $(\mathrm{Nu})$

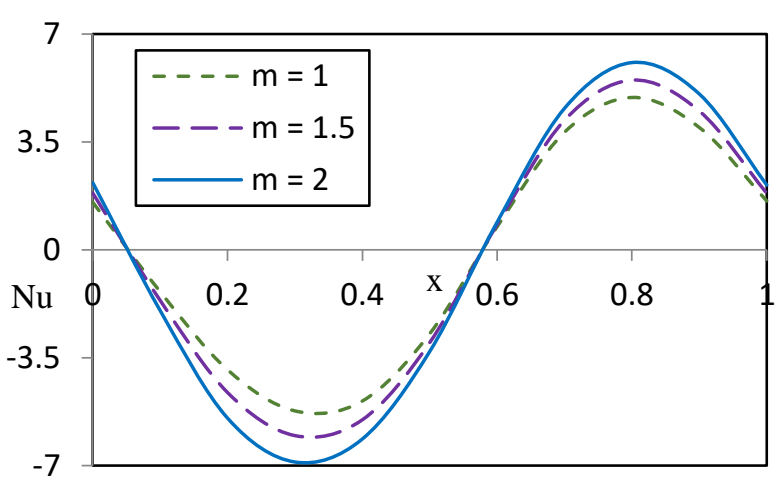

Figure 5(c). Impact of $\mathrm{m}$ on Nusselt number $(\mathrm{Nu})$

Fig. 6 is displayed for the influence of hartmann number, radiation parameter, schmidt number, soret number and chemical reaction parameter on the variation of mass transfer rate (Sherwood number (Sh)). The following default parameter values are adopted for computations: $\mathrm{Da}=0.1, \mathrm{M}$ $=2, \mathrm{~N}=0.3, \mathrm{Pr}=5, \mathrm{~m}=1, \mathrm{Sc}=0.3, \mathrm{Sr}=3, \mathrm{~S}=1.5 \eta=0.5, \beta$ $=0.7, \mathrm{Br}=0.3, v=0.1, \phi=\pi / 6, \alpha=\pi / 6, \mathrm{k}_{1}=0.1, \mathrm{p}=-1, \mathrm{t}=$ $0.4, \varepsilon=0.2$. We notice from these graphs $6(\mathrm{a})-6(\mathrm{c})$ that the absolute value of mass transfer rate (Sherwood number Sh) enhances in the region $x \in[0.05,0.57]$ while it reduces in the rest of the region $x \in[0,0.05] \mathrm{U}[0.57,1]$ by increase in radiation parameter $(\mathrm{N}=0.3,0.5,0.7)$, schmidt number $(\mathrm{Sc}=$ $0.1,0.3,0.5)$ and soret number $(\mathrm{Sr}=1,3,5)$. Fig. $6(\mathrm{~d})$ reveals the effect of chemical reaction on Sherwood number (Sh). It is interesting to note that the absolute value of sherwood number (Sh) enhances in the region $\mathrm{x} \in[0,0.05] \mathrm{U}[0.57,1]$ while it reduces in the rest of the region $\mathrm{x} \in[0.05,0.57]$ with ascending values of chemical reaction parameter $(\mathrm{S}=1.5,3$, 
4.5). We, conclude from the graphs that the sherwood number (Sh) is in an oscillatory behaviour, which may be due to peristalsis.

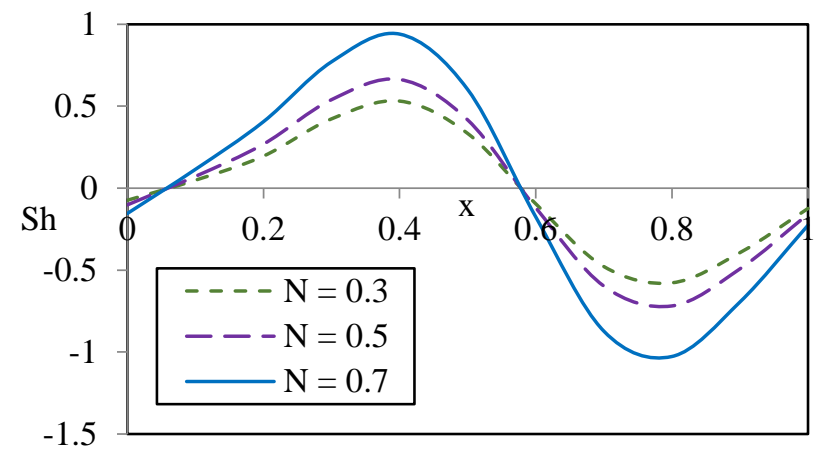

Figure 6(a). Impact of N on Sherwood number (Sh)

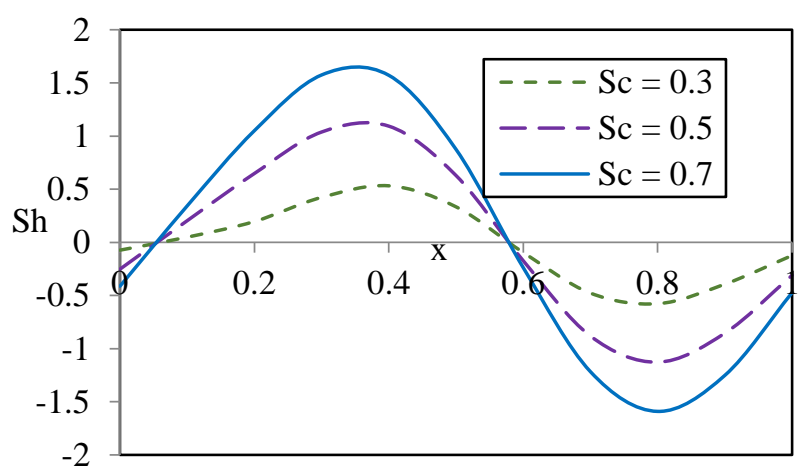

Figure 6(b). Impact of Sc on Sherwood number (Sh)

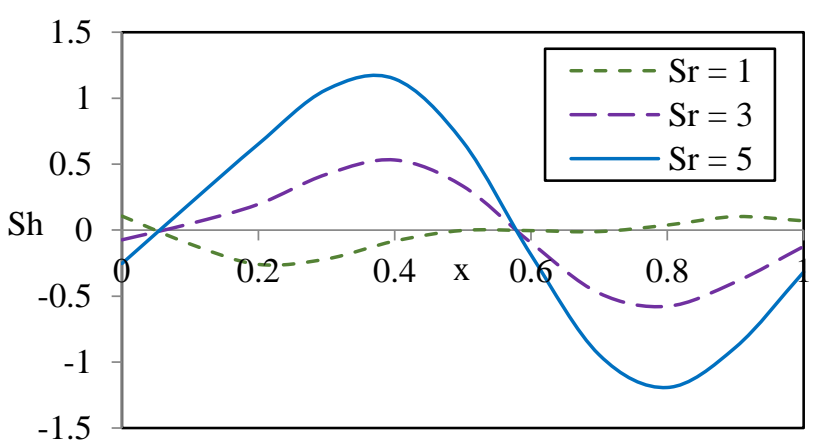

Figure 6(c). Impact of Sr on Sherwood number (Sh)

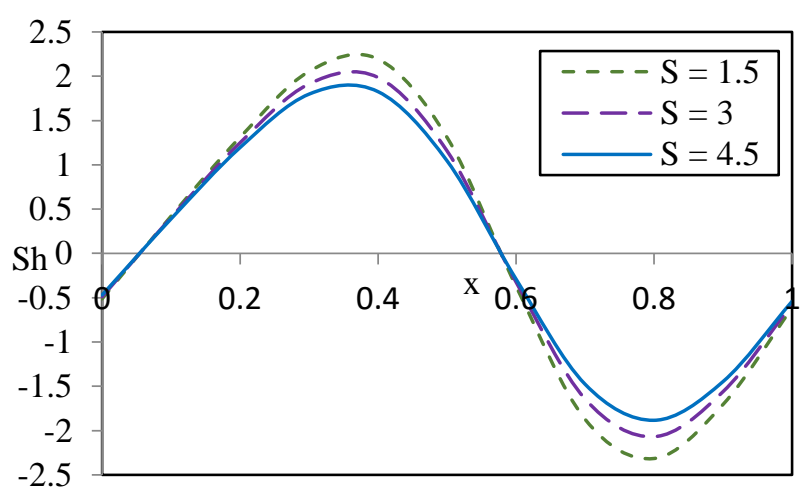

Figure 6(d). Impact of S on Sherwood number (Sh)

\section{CONCLUSIONS}

The current study addressed the hall current, radiation and velocity slip on hydromagnetic physiological hemodynamic fluid with joule heating and mass transfer in presence of chemical reaction in an inclined asymmetrical tapered vertical channel have been investigated. The main observations of this work are cited below.

- The pressure gradient $(\mathrm{dp} / \mathrm{dx})$ reduces with the increase of porosity parameter $(\mathrm{Da})$, hall parameter $(\mathrm{m})$ and volumetric flow rate $(\bar{Q})$ while it increases by increasing hartmann number $(\mathrm{M})$, gravitational parameter $(\eta)$ and slip parameter $(\beta)$.

- $\quad$ The temperature of the fluid rises with an increase in hartmann number $(\mathrm{M})$, porosity parameter $(\mathrm{Da})$, radiation parameter $(\mathrm{N})$, prandtl number $(\mathrm{Pr})$, brinkman number $(\mathrm{Br})$, heat source parameter $(v)$ and hall current parameter $(\mathrm{m})$ while it decreases by increase of slip parameter $(\beta)$.

- Concentration profile reduces with increase in hartmann number $(\mathrm{M})$, porosity parameter $(\mathrm{Da})$, radiation parameter $(\mathrm{N})$, prandtl number $(\mathrm{Pr})$, brinkman number $(\mathrm{Br})$, heat source parameter $(v)$ hall current parameter $(\mathrm{m})$, schmidt number (Sc) and soret number $(\mathrm{Sr})$.

- The concentration profile reduces with a rise in chemical reaction parameter $(S)$.

- An absolute value of nusselt number enhances in the region $x \in[0,0.05] \mathrm{U}[0.57,1]$ while it reduces in the rest of the region $x \in[0.05,0.57]$ by increase in hartmann number $(\mathrm{M})$, radiation parameter $(\mathrm{N})$, brinkman number $(\mathrm{Br})$ and hall current parameter $(\mathrm{m})$.

- An absolute value of nusselt number enhances in the region $x \in[0,0.05] \mathrm{U}[0.57,1]$ while it reduces in the rest of the region $x \in[0.05,0.57]$ by increase in hartmann number $(\mathrm{M})$, radiation parameter $(\mathrm{N})$, brinkman number $(\mathrm{Br})$ and hall current parameter $(\mathrm{m})$.

- Mass transfer rate (Sherwood number Sh) enhances in the region $x \in[0.05,0.57]$ while it reduces in the rest of the region $x \in[0,0.05] \mathrm{U}[0.57,1]$ by increasing hartmann number $(\mathrm{M})$, radiation parameter $(\mathrm{N})$, schmidt number $(\mathrm{Sc})$ and soret number $(\mathrm{Sr})$.

- An effect of chemical reaction on $\mathrm{Sh}$ is quite opposite to that of hartmann number $(\mathrm{M})$, radiation parameter $(\mathrm{N})$, schmidt number $(\mathrm{Sc})$ and soret number $(\mathrm{Sr})$.

\section{ACKNOWLEDGEMENTS}

We would like to thank the anonymous reviewers and the editor for their encouraging comments and constructive suggestions in improving the manuscript of the present study.

\section{REFERENCES}

[1] Latham TW. (1966). Fluid motion in a peristaltic pump. M.Sc Thesis. Massachusetts Institute of Technology, Cambridge, MA.

[2] Shapiro AH, Jaffrin MY, Wienberg SL. (1969). Peristaltic pumping with long wavelengths at low Reynolds number. J. Fluid Mech 37: 799-825. https:// doi.org/10.1017/S0022112069000899

[3] Abd Elmaboud Y, Mekheimer Kh S. (2011). Non-linear peristaltic transport of a second-order fluid through a porous medium. Applied Mathematical Modelling 35: 2695-2710. https://doi.org/10.1016/ j.apm.2010.11.031

[4] Mekheimer KhS, Salem AM., Zaher AZ. (2014). Peristaltically induced MHD slip flow in a porous 
medium due to a surface acoustic wavy wall. Journal of the Egyptian Mathematical Society 2: 143-151. https:// doi.org/10.1016/j.joems.2013.05.003

[5] Mekheimer Kh S, Abd Elmaboud Y, Abdellateef AI. (2013). Particulate suspension flow induced by sinusoidal peristaltic waves through eccentric cylinders: Thread annular. Int. J. Biomath 06:1 350026. https:// doi.org/10.1142/S1793524513500265

[6] Hayat T, Abbasi FM, Alsaedi A. (2014). Soret and Dufour effects on peristaltic flow in an asymmetric channel. Arab. J. Sci. Eng 39: 4341-4349. https://doi. org/10.1007/s13369-014-1163-y

[7] Pandey SK, Tripathi D. (2012). Unsteady peristaltic transport of Maxwell fluid through finite length tube: Application to esophageal swallowing. Appl. Math. Mech 33: 15-24. https://doi.org/10.1007/s10483-0121530-9

[8] Tripathi D, Ali N, Hayat T, Chuabe MK, Hendi AA. (2011).Peristaltic flow of MHD Jeffrey fluid through finite length cylindrical tube. Appl. Math. Mech 32: 1231-1244. https://doi.org/10.1007/s10483-011-14967

[9] Srinivas S, Kothandapani M. (2009). The influence of heat and mass transfer on MHD peristaltic flow through a porous space with compliant walls. Appl. Math. Comput 213: 197-208. https://doi.org/10.1016/j.amc. 2009.02.054

[10] Lakshminarayana P, Sreenadh S, Sucharitha G. (2013). Influence of heat transfer on MHD peristaltic flow through a vertical asymmetric porous channel. Elixir Appl. Math 54: 12413-12419. https://doi.org/10.18869/ 10.18869/acadpub.jafm.68.235.24709

[11] Vajravelu K, Sreenadh S, Lakshminarayana P. (2011). The influence of heat transfer on peristaltic transport of a jeffrey fluid in a vertical porous stratum. Commun. Non-linear Sci. Numer. Simulat. 16: 3107-3125. https:// doi.org/10.1016/j.cnsns.2010.11.001

[12] Agarwal RP. (1989). Heat transfer to pulsatile flow of conducting fluid in a porous channel. Ph.D Thesis, Allahabad.

[13] Tang Dalin, Shen MC. (1989). Peristaltic transport of a heat-conducting fluid subject to Newton's cooling law at the boundary. Int. J. Eng. Sci 27: 809-825. https://doi.org/10.1016/0020-7225(89)90047-5

[14] Radhakrishnamacharya G, Srinivasulu CH. (2007). Influence of wall properties on peristaltic transport with heat transfer. $\mathrm{C} \quad \mathrm{R}$ Mecanique 335: 369-373. https://doi.org/10.1016/j.crme.2007.05.002

[15] Abbasi FM, Saba Shehzad SA. (2017). Heat transfer analysis for peristaltic flow of carreau-Yasuda fluid through a curved channel with radial magnetic field. International journal of Heat and Mass Transfer 115: 777-783. https://doi.org/10.1016/j.ijheatmasstransfer.2017.08.048

[16] Hayat T, Nisar Z, Ahmad B, Yasmin H. (2015) Simultaneous effects of slip and wall properties on MHD peristaltic motion of nanofluid with joule heating. Journal of Magnetism and Magnetic Materials 395: 4858. https://doi.org/10.1016/j.jmmm.2015.07.027

[17] Hayat T, Abbasi FM, Al-Yami M, Monaquel S. (2014). Slip and joule heating effects in mixed convection peristaltic transport of nanofluid with Soret and Dufour effects. J. Mol. Liq 194: 93-99. https://doi.org/10.1016/j.molliq.2014.01.021
[18] Mekheimer Kh S, Husseny SZA, Abd Elmaboud Y. (2010). Effects of heat transfer and space porosity on peristaltic flow in a vertical asymmetric channel. Numer. Meth. Partial Diff. Eqn 26: 747-770. https://doi.org/10.1002/num.20451

[19] Srinivas S, Gayathri R, Kothandapani M. (2011). Mixed convective heat and mass transfer in an asymmetric channel with peristalsis. Communications in Nonlinear Science and Numerical Simulation 16: 1845-1862. https://doi.org/10.1016/j.cnsns.2010.08.004

[20] Eldabe NT, Elogail MA, Elshaboury SM, Alfaisal A. Hasan. (2016). Hall effects on the peristaltic transport of williamson fluid through a porous medium with heat and mass transfer. Applied Mathematical Modelling 40: 315-328. https://doi.org/10.1016/j.apm.2015.04.043

[21] Shehzad SA, Abbasi FM, Hayat T, Alsaadi F. (2014). MHD mixed convective peristaltic motion of nanofluid with joule heating and thermophoresis effects. PLoS ONE 9(11): e111417. https://doi.org/10.1371/journal. pone.0111417

[22] Ravikumar S. (2015). Effect of couple stress fluid flow on magnetohydrodynamic peristaltic blood flow with porous medium trough inclined channel in the presence of slip effect-Blood flow study. International Journal of Bio-Science and Bio-Technology 7(5): 65-84. https:// doi.org/10.14257/ijbsbt.2015.7.5.07

[23] Ravikumar S. (2016). Analysis of heat transfer on mhd peristaltic blood flow with porous medium through coaxial vertical tapered asymmetric channel with radiation-Blood flow study. International Journal of Bio-Science and Bio-Technology 8(2): 395-408. https://doi.org/10.14257/ijbsbt.2016.8.2.28

[24] Ravikumar S. (2016). Hydromagnetic peristaltic transportation with porous medium through coaxial asymmetric vertical tapered channel and joule heating. Applications and Applied Mathematics: An International Journal (AAM) 11: 735-747.

[25] Misra JC, Adhikary SD. (2016). MHD oscillatory channel flow, heat and mass transfer in a physiological fluid in presence of chemical reaction. Alexandria Engineering Journal 55: 287-297. https://doi.org/ 10.1016/j.aej.2015.10.005

[26] Ravi Kumar S, Abzal SK. (2017). Combined influence of hall currents and joule heating on hemodynamic peristaltic flow with porous medium through a vertical tapered asymmetric channel with radiation. Frontiers in Heat and Mass Transfer (FHMT) 9(19): 1-9. http://dx.doi.org/10.5098/hmt.9.19

[27] Ameer Ahamad N, Ravikumar S, Kalimuthu Govindaraju. (2017). Influence of radiation on MHD peristaltic blood flow through a tapered channel in presence of slip and joule heating. AIP Conference Proceedings 1863: 560091-1-560091-5. http://doi.org/ $10.1063 / 1.4992774$

[28] Darbhasayanam Srinivasacharya, Shafeeurrahman Md. (2017). Joule heating effect on entropy generation in MHD mixed convection flow of chemically reacting nanofluid between two concentric cylinders. International Journal of Heat and Technology 35: 487497. https://doi.org/10.18280/ijht.350305

[29] Srinivas S, Kothandapani M. (2008). Peristaltic transport in an asymmetric channel with heat transfer - a note. International Communications in Heat and Mass 
$\operatorname{Re}$

https://doi.org/10.1016/j.icheatmasstransfer.2007.08.01

[30] Cogley ACL, Vinvent WG, Giles ES. (1968).

Differential approximation for radiative heat transfer in non-linear equations-grey gas near equilibrium. American Institute of Aeronautics and Astronautics 6: 551-553.

[31] Kothandapani M, Prakash J, Pushparaj V. (2015). Effects of heat transfer, magnetic field and space porosity on peristaltic flow of a Newtonian fluid in a tapered channel. Applied Mechanics and Materials 813814:

679-684.

https://doi.org/10.4028/www.scientific.net/AMM.813814.679

\section{NOMENCLATURE}

u, v

$\mathrm{x}, \mathrm{y}$

b

d

c

$g$

$m^{\prime}$

$\mathrm{t}$

$\mathrm{B}_{0}$

$\mathrm{p}$

T,C

$k$

K

$\mathrm{k}_{1}$

$Q_{0}$

$C_{p}$

$k_{2}$ velocity components (unit: $\mathrm{m} / \mathrm{s}$ )

cartesian coordinates (unit: $\mathrm{m}$ )

width of the channel

wave amplitude (unit: m)

ave velocity (unit: $\mathrm{m} / \mathrm{s}$ )

acceleration due to gravity

non-uniform parameter

time (unit:s)

magnetic field (unit:T)

pressure in fixed frame (unit: $\mathrm{N} / \mathrm{m}^{2}$ )

temperature (unit:K) and concentration

thermal conductivity (uniy:W/(m.k))

permeability parameter

permeability of the porous medium

heat addition/absorption

specific heat at constant pressure

rate of chemical reaction

\section{Greek symbols}

$\rho$
$\delta$
$\eta, \eta$
$\sigma$
$\phi$
$\mu$
$\lambda$
$\alpha$
$\nu$
$\beta$
$\theta$
$\Phi$

reynolds number volumetric flow rate (unit: $\mathrm{m}^{3} / \mathrm{s}$ ) hartman number porosity parameter eckert number brinkman number schmidt number soret number hall parameter chemical reaction parameter radiation parameter mean temperature mass diffusivity (unit: $\mathrm{m}^{2} / \mathrm{s}$ ) thermal diffusion ratio nusselt number sherwood number velocity prandtl number

density of the fluid (unit: $\mathrm{kg} / \mathrm{m}^{3}$ )

wave number (unit: $\mathrm{m}^{-1}$ ) gravitational parameters electrical conductivity (unit: $\mathrm{S} / \mathrm{m}$ ) phase shif viscosity of the fluid (unit: N.s $/ \mathrm{m}^{2}$ ) wavelength (unit: m) inclination of the channel heat source/sink parameter slip parameter dimensionless temperature dimensionless concentration 